\title{
Contaminación agricolaación por uso de aguas residuales
}

\author{
Agricultural pollution from wastewater use \\ Poluição agrícola pelo uso de águas residuais
}

\author{
- Juan de Dios Aguilar Sánchez \\ juasajae@gmail.com \\ ORCID: 0000-0001-5152-5665
}

\author{
- Napoleón Cubas Irigoin \\ napochota1@gmail.com \\ ORCID: 0000-0001-5950-7525
}

\section{Universidad Nacional Autónoma de Chota, Chota-Perú}

\section{RESUMEN}

El objetivo de esta investigación fue mostrar el índice de contaminación de la producción agrícola por el uso de las aguas residuales. La metodología empleada fue una investigación experimental, de campo documental y descriptiva, se tomaron muestras de agua residual de las cuencas formadas por las quebradas San Mateo, Colpamayo, San Juan y Río Chotano. Se analizaron y se observó que las aguas residuales no llenan los requisitos adecuados para ser usado para riego. Se tomaron además muestras de verduras y hortalizas regadas con esas aguas y se les detecto contaminación por presencia de coliformes, estreptococos y salmonella. Como conclusión se puede mencionar que Se lograron identificar los parámetros físicos y de agregación para las aguas residuales sin tratamiento de quebradas San Mateo, Colpamayo, San Juan y Río Chotano provenientes de la ciudad de Chota, donde se establece que solo dos parámetros: el $\mathrm{pH}$ y los sólidos en suspendidos totales están entre los valores adecuados. La temperatura y la turbidez están fuera de rango por exceso en sus valores y el oxígeno disuelto, la conductividad eléctrica, los sólidos disueltos totales están fuera de rango por no alcanzar el mínimo valor. Entre los parámetros inorgánicos no metálicos que están en los valores aceptables están: la alcalinidad, los cloruros, la dureza, los nitratos, los nitritos, los sulfatos y la demanda química de oxígeno y quienes no están en los valores correctos son: los fosfatos, el amonio, y la demanda química de oxígeno.

Palabras clave: Aguas Residuales; Contaminación; Muestras, Coliformes
ABSTRACT

RESUMO
The objective of this research was to show the pollution index of agricultural production due to the use of wastewater. The methodology used was an experimental, documentary and descriptive field investigation, samples of residual water were taken from the basins formed by the San Mateo, Colpamayo, San Juan and Río Chotano streams. They were analyzed and it was observed that the wastewater does not meet the adequate requirements to be used for irrigation. Samples of vegetables watered with these waters were also taken and contamination was detected due to the presence of coliforms, streptococci and salmonella. As a conclusion, it can be mentioned that the physical and aggregation parameters for the untreated wastewater from the San Mateo, Colpamayo, San Juan and Río Chotano streams from the city of Chota were identified, where it is established that only two parameters: the $\mathrm{pH}$ and the solids in total suspended are between the suitable values. Temperature and turbidity are out of range due to excess values and dissolved oxygen, electrical conductivity; total dissolved solids are out of range due to not reaching the minimum value. Among the non-metallic inorganic parameters that are in the acceptable values are: alkalinity, chlorides, hardness, nitrates, nitrites, sulfates and chemical oxygen demand and those who are not in the correct values are: phosphates, ammonium, and chemical oxygen demand.

Key words: Wastewater; Pollution; Samples; Coliforms
0 objetivo desta pesquisa foi mostrar o índice de poluição da produção agrícola devido ao uso de águas residuárias. A metodologia utilizada foi uma investigação experimental, documental e descritiva de campo, foram retiradas amostras de águas residuais das bacias formadas pelos riachos San Mateo, Colpamayo, San Juan e Río Chotano. Eles foram analisados e observou-se que a água residuária não atende aos requisitos adequados para ser utilizada na irrigação. Também foram colhidas amostras de hortaliças regadas com essas águas e detectada contaminação pela presença de coliformes, estreptococos e salmonelas. Como conclusão, pode-se citar que foram identificados os parâmetros físicos e de agregação das águas residuais não tratadas dos riachos San Mateo, Colpamayo, San Juan e Río Chotano da cidade de Chota, onde se estabelece que apenas dois parâmetros: o pH e os sólidos em suspensão total estão entre os valores adequados. A temperatura e a turbidez estão fora da faixa devido a valores excessivos e o oxigênio dissolvido, a condutividade elétrica e o total de sólidos dissolvidos estão fora da faixa por não atingir o valor mínimo. Entre os parâmetros inorgânicos não metálicos que estão nos valores aceitáveis estão: alcalinidade, cloretos, dureza, nitratos, nitritos, sulfatos e demanda química de oxigênio e aqueles que não estão nos valores corretos estão: fosfatos, amônio e demanda de oxigênio químico.

Palavras-chave: Água Residual; Poluição; Amostras, coliformes 


\section{INTRODUCCIÓN}

El uso en la agricultura de aguas residuales es una opción cada vez más utilizada a nivel mundial, (1) el agua es un recurso escaso y además valioso, pero esta debe de cumplir con unos parámetros para que su uso no incida en la calidad de los productos obtenidos, pues el riego con agua contaminada de alimentos es muy peligroso para el hombre y para los suelos. Para Zamora, et. al. (2) El uso de las aguas residuales en la agricultura, puede aumentar el ingreso de materias orgánicas y nutrientes a los suelos cultivados lo cual contribuye a mantener e incrementar la fertilidad del mismo, pero también puede causar efectos ambientales nocivos que deterioran la calidad del suelo.

La contaminación de productos agrícolas como verduras y hortalizas por el uso de riego de aguas residuales en su producción se debe en gran parte por la omisión de políticas públicas de los gobernantes, que deben velar por que se utilicen solo aguas aptas para el riego, deben de dotar la infraestructura para el adecuado tratamiento y manejo de las aguas residuales, por otro lado tenemos a los agricultores que por falta de información o irresponsabilidad utilizan estas aguas, y por parte de los consumidores que son el eslabón más débil de la cadena, por no observar normas sanitarias adecuadas al momento de consumir estos productos.

En América Latina, la contaminación de la producción agrícola es por efecto del uso aguas residuales provenientes de las ciudades, actividad minera, agrícola y la acumulación de residuos sólidos, que son llevados a través del riego y la descomposición es absorbida por los diversos cultivos que se contaminan con los compuestos químicos y bacterias. Peña, Ducci, y Zamora, (3) Resaltan que:

Las diferentes actividades productivas al generar desechos diversos, son las fuentes principales de contaminación de los diferentes cuerpos de agua; lo que se traduce en la desaparición de la vegetación natural, así como en la muerte de peces y demás animales acuáticos. (p. 15).

El uso de aguas residuales en la agricultura contamina la producción agrícola, constituyendo un riesgo para la salud de los consumidores.

Los principales valles de ríos y quebradas en Perú se ven afectados por el desarrollo urbano y demográfico que no solo ocupan los terrenos de cultivo, sino que los contaminan con aguas servidas y desagües que en los valles aledaños son utilizados en el riego de productos agrícolas. Para el Ministerio de Agricultura, (4) a través de La Autoridad Nacional de Agua (ANA) indica que el río Rímac, uno de los principales proveedores del recurso hídrico a Lima Metropolitana está enfermo, en su recorrido desde los andes hasta su desembocadura en el mar se han identificado más 1185 puntos de contaminación, en la cuenca alta el río recibe aguas acidas de industrias y relaves mineros; en la cuenca media el río se ve afectado por la contaminación de la actividad agrícola, mientras que la cuenca baja por los residuos sólidos, químicos de la industria y desagües. El diagnóstico hecho por el ANA señala que para descontaminar el río tendrían que transcurrir unos 10 años, demandando una inversión de mil millones de dólares, para construir plantas de tratamiento de aguas, 
residuos sólidos y programas de reforestación.

En la región Cajamarca, la mayoría de los valles de quebradas y ríos reciben los efectos contaminantes de desagües de viviendas, que luego son utilizadas en la agricultura sin ningún tratamiento. El diario de Noticias Ser, (5) informa que en Cajamarca diariamente se produce 194 litros de aguas residuales por segundo, van a la cuenca del río Mashcón y Chonta y lo utilizan para la agricultura. Los sólidos al interior de las aguas van produciendo peligrosos compuestos químicos, mientras que las aguas residuales van concentrando coliformes" (párr. 1). Se demuestra que las quebradas y ríos recolectan residuos sólidos y aguas residuales que no solo afectan a las aguas naturales, sino que desaparece la flora y fauna y contamina la producción agrícola.

No obstante el uso sin tratamiento previo del agua puede ocasionar problemas (...), contaminación con metales pesados y la presencia de algunos organismos patógenos al hombre. Por ello es necesario evaluar su uso cuantificando su efecto sobre las propiedades químicas del suelo con el fin de garantizar que la utilización no acarre problemas ambientales, ni de salud pública (2).

Las quebradas y ríos aledaños a los centros poblados dentro de la región corren con la misma suerte, son contaminados con relaves mineros, como el caso del río Llaucano que recibe las aguas residuales de la minera Golfi y los desagües de los poblados de Hualgayoc y Bambamarca o Yanacocha que según el diario La República, (6) de fecha 17 de octubre vierte más de 40 millones de metros cúbicos de aguas residuales al ambiente y contaminan directamente a ríos y quebradas aledañas: quebrada Honda, río Chonta, río Porcón y Río Rejo, advirtiendo que tendrían que pasar unos 50 años para recuperar estas aguas del manto freático y están contaminando la producción agrícola y ganadera.

La contaminación de las aguas de quebradas y ríos en los distintos distritos de la provincia de Chota, proviene de los desagües, las redes vierten sus aguas a quebradas y ríos. La ciudad de Chota se desarrolla entre las cuencas de quebradas San Mateo, Colpamayo, San Juan y Río Chotano, cuyo caudal mayoritariamente proviene de las aguas residuales de la ciudad que desembocan en el río, en los valles de las quebradas y el río existen terrenos de cultivo que utilizan las aguas provenientes de los desagües para la irrigación de la producción agrícola, cuyo excedente se vende en el mercado local.

Para la realización de esta investigación se utilizaron como referentes los estudios Forsi, (7), señala que los agricultores que utilizan aguas residuales no tratadas en los cultivos trae efectos negativos para la salud, el reutilizar las aguas residuales favorece al desarrollo socioeconómico, debido que al ser tratadas se incrementa las áreas de cultivo, generando trabajo y beneficio económico. Guadarrama y Galván, (8) Resaltan que con el crecimiento de la población y el desarrollo industrial se sobreexplota el agua y el suelo propiciando su contaminación a través de la proliferación de aguas residuales y residuos sólidos que lamentablemente contaminan el campo agrícola, habiendo entre unas 20 a 25 millones de hectáreas de terrenos que son irrigados con aguas residuales, las cosechas van entre 18 a 22 millones de hectáreas al año, esta producción consumida de diversas 
maneras, no tiene el control sanitario. Peña et al. (3), asegura que las aguas residuales producidas en las zonas urbanas y rurales, se conduzcan sin ningún problema a las plantas de tratamiento, para ello los diversos hogares deben tener acceso a redes de desagüe para evitar la contaminación de la producción agrícola. Mendoza (9), en la Evaluación fisicoquímica de la calidad del agua superficial en el Centro Poblado de Sacsamarca, región Ayacucho, encuentra que los fosfatos presentan $1.51 \mathrm{ppm}$, el arsénico $0.13 \mathrm{ppm}$, demuestra que hay una importante concentración de arsenio en el río Caracha, y significa una alerta para las autoridades porque el parámetro es un indicador de eutrofización y contaminación de aguas. Tarrillo (10) señala que resulta importante conocer la cantidad componentes físicos y químicos, la microbiología que se desplaza por las redes de desagüe y los ríos a fin de evitar el uso en la agricultura. Reyes (11), destaca que los parámetros fisicoquímicos que se investigaron in situ del caudal de agua del río Chillón, proviene de aguas superficiales no reguladas, agua superficial regulada, agua subterránea y agua de recuperación, pueden generar un peligro físico natural en la zona agrícola que se utilice.

El estudio de la contaminación de la producción agrícola por efecto del uso de las aguas residuales sin tratamiento previo, permitirá que todos los involucrados se sensibilicen, reflexionen y emprendan los correctivos necesarios como puede ser la construcción de una planta de tratamiento de aguas residuales, para evitar que los efluentes vayan a las quebradas y ríos, contaminando las aguas naturales. Se evite la degradación los suelos afectando al ambiente, y como fin último obtener una producción sana para la sostenibilidad y mejorar la calidad de vida de la población consumidora.

Para Galindo, et al. (12) El uso de agua residual tratada de origen urbano para irrigar suelos agrícolas destinados a la producción de forrajes es una alternativa que favorece el aporte de nutrientes al cultivo y minimiza la contaminación en los sitios de descarga; sin embargo, se requiere conocimiento acerca de la calidad de esta agua. Por lo tanto, es necesario caracterización de las aguas residuales.

La investigación tuvo como objetivo general: Mostrar el índice de contaminación de la producción agrícola por el uso de las aguas residuales, y como objetivos específicos: identificar los parámetros físicos y de agregación; parámetros inorgánicos no metálicos, agentes contaminantes de las aguas residuales y determinar la acumulación de agentes contaminantes en la producción agrícola por el uso de las aguas residuales. La metodología del diseño experimental se basó en un estudio cuasiexperimental en el que se evaluaron los efectos contaminantes del riego con aguas residuales de cultivos de repollo, papa, racacha, lechuga, acelga, cebolla y berenjena. En los valles de las quebradas, San Mateo, Colpa Mayo, San Juan y Río Chotano, ubicados en los alrededores de la ciudad de Chota, departamento de Cajamarca. Para el análisis e interpretación de los resultados se utilizaron la guía normas de calidad del agua de la Organización Mundial de la Salud (13), las normas de inocuidad de los alimentos de la Organización Mundial de la Salud (14) los indicadores de calidad del país y de Organización de las Naciones Unidas para la Alimentación (15), la Resolución Ministerial No 323 - 2015-MINAM (16), los indicadores 
proporcionados por la Dirección General de Salud $(17,18)$, y D. S. N 015-2015-MINAM (16).

\section{METODOLOGÍA}

El trabajo se desarrolló en los valles de las quebradas, San Mateo, Colpa Mayo, San Juan y Río Chotano, ubicados en los alrededores de la ciudad de Chota, departamento de Cajamarca, el área de estudio partió del primer punto muestral a 2388 m.s.n.m. en la quebrada Colpa Mayo, hasta los 2302 m.s.n.m., en la unión con el Río Chotano, siguiendo el curso de $3 \mathrm{Km}$., hasta los 2257 m.s.n.m. en la desembocadura de la quebrada San Mateo, continuando el recorrido aguas arriba hasta los 2412 m.s.n.m., en el punto de encuentro con las aguas de la quebrada San Juan a 2360 m.s.n.m. recorriendo hasta los 2289 m.s.n.m.; la distancia total es de $10.03 \mathrm{Km}$.

Los materiales utilizados fueron: 01 estación total, 02 prismas, 01 GPS satelital, 01 wincha de 5 metros, una brújula, 01 corrector, movilidad, esmalte y brochas para marcar los puntos de muestra de la producción de repollo, papa, racacha, lechuga, acelga, cebolla y berenjena. Para las muestras incitas del agua residual se utilizó un PH-METRO. Para recolección de muestras de las aguas residuales frascos con tapa graduados. El levantamiento topográfico se hizo ubicando los puntos de muestreo de las aguas residuales, se estableció un radio de 50 por 30 metros de los terrenos de cultivo.

El desarrollo del trabajo, consistió en hacer el análisis físico, de agregación, parámetros inorgánicos no metálicos $\mathrm{y}$ agentes contaminantes de las aguas residuales y el análisis microbiológico a la producción agrícola (repollo, papa, racacha, lechuga, acelga, cebolla y berenjena ) para determinar el índice de contaminación en los valles de las quebradas, San Mateo, Colpa Mayo, San Juan y Río Chotano, los resultados de las muestras se compararon con los estándares nacionales e internacionales que plantea la Organización Mundial de la Salud (13), para determinar la contaminación de la producción agrícola se optó por hacer el análisis microbiológico de hortalizas, los resultados se compararon con la norma sanitaria que establece los criterios microbiológicos de calidad sanitaria e inocuidad para los alimentos y bebidas de consumo humano (16) la Resolución Ministerial $\mathrm{N}^{\circ} 323$ - 2015-MINAM (16) y la OMS (13).

La población en estudio estuvo constituida por colectores de aguas residuales que desembocan en las quebradas San Mateo, Colpa Mayo, San Juan y río Chotano, los productos agrícolas (hortalizas) que se produce en las parcelas irrigadas con las aguas residuales en un área de 50 por 30 metros por cada punto crítico a fin de conocer la calidad toxológica en la producción agrícola.

La muestra fue seleccionada intencionalmente y estuvo representada por los 17 puntos críticos de desemboque de aguas residuales en las quebradas y ríos indicados, las muestras de productos agrícolas (hortalizas y verduras) se tomaron en función de los 9 puntos cuyas aguas se presumen están más contaminadas y se utilizan para irrigar sembríos de tubérculos y verduras en una malla de 50 por 30 metros.

La técnica de recolección de datos fue la observación para la identificación de los puntos críticos, la recolección de muestras, hacer el análisis y registro de resultados. 
Como instrumento se utilizó la ficha de registro donde se anotaron los resultados, se analizaron y compararon con las normas emitidas por la Resolución Ministerial № 323 - 2015-MINAM y DIGESA, la FAO (15), la OMS (13), DIGESA (18).

\section{RESULTADOS Y DISCUSIÓN}

Se tomaron un total de 17 muestras de agua en varios sitios del área objeto de estudio y se realizaron estudios para estudiar los parámetros físicos y de agregación que se presentan en la tabla 1 que se muestra a continuación.

Tabla 1. Parámetros físicos y de agregación de las aguas residuales.

\begin{tabular}{|c|c|c|c|c|c|c|c|c|}
\hline \multirow[b]{2}{*}{$\begin{array}{l}\text { Quebrada/ } \\
\text { Ríos }\end{array}$} & \multirow[b]{2}{*}{ Muestra } & \multicolumn{7}{|c|}{ PARÁMETROS FÍSICOS Y DE AGREGACIÓN } \\
\hline & & pH & $\mathrm{T}^{\circ} 0$ & Turbidez & $\begin{array}{l}\text { Oxígeno } \\
\text { disuelto }\end{array}$ & $\begin{array}{c}\text { Conductividad } \\
\text { eléctrica }\end{array}$ & $\begin{array}{c}\text { Solidos } \\
\text { disueltos } \\
\text { totales } \\
\end{array}$ & $\begin{array}{c}\text { Solidos } \\
\text { suspendidos } \\
\text { totales } \\
\end{array}$ \\
\hline \multirow{5}{*}{ Colpa Mayo } & M1 p1 & 7.84 & 17.3 & 1122 & 1.65 & 748.0 & 448.8 & 1.496 \\
\hline & M2 p2 & 7.89 & 17.5 & 930 & 1.70 & 620.0 & 372.0 & 1.439 \\
\hline & M3 p3 & 7.12 & 17.99 & 1506 & 1,77 & 1005.0 & 603.0 & 1.774 \\
\hline & M4 p4 & 7.87 & 17.72 & 897 & 1.87 & 598.0 & 358.8 & 1.533 \\
\hline & M5 p5 & 7,71 & 17.31 & 1062 & 1.95 & 708.0 & 424.8 & 0.134 \\
\hline \multirow{7}{*}{$\begin{array}{c}\text { Río } \\
\text { Chotano }\end{array}$} & M6 p6 & 7.57 & 17.51 & 1179 & 1.96 & 787.0 & 472.2 & 0.356 \\
\hline & M7 p7 & 7.00 & 17.62 & 1134 & 1.85 & 765.0 & 459.0 & 0.391 \\
\hline & M8 p8 & 7.88 & 17.92 & 951 & 1.95 & 634.0 & 380.4 & 1.703 \\
\hline & M9 p9 & 7.88 & 17.23 & 591 & 1.96 & 394.0 & 236.4 & 0.307 \\
\hline & M10 p10 & 7.82 & 17.28 & 702 & 1.84 & 389.0 & 233.4 & 1.046 \\
\hline & M11 p11 & 7.81 & 17.32 & 795 & 1.82 & 435.0 & 261.0 & 0.435 \\
\hline & M12 p12 & 7.84 & 17,53 & 732 & 2.02 & 488.0 & 292.8 & 0.375 \\
\hline \multirow{3}{*}{ San Juan } & M13 p13 & 7.22 & 17.41 & 1239 & 2.01 & 826.0 & 495.6 & 0.587 \\
\hline & M14 p14 & 7.35 & 17.97 & 1161 & 2.07 & 774.0 & 664.4 & 0.415 \\
\hline & M15 p15 & 7.26 & 17.8 & 1026 & 2.07 & 684.0 & 410.4 & 0.552 \\
\hline \multirow[t]{2}{*}{ San Mateo } & M16 p16 & 7.35 & 1899 & 1404 & 1.98 & 937.0 & 562.2 & 0.584 \\
\hline & M17 p17 & 7.42 & 17.85 & 1311 & 1.93 & 879.0 & 527.4 & 1.020 \\
\hline
\end{tabular}

Fuente: Informe de ensayos 335 - 352.

Al comparar los resultados de los ensayos de la muestra con las normas regulatorias se obtuvieron los siguientes resultados.

El potencial de Hidrógeno $(\mathrm{pH})$ de $7.00 \mathrm{a}$ 7.89 cumpliendo con el parámetro de la norma de 6.5 a 8.5 unidades de $\mathrm{pH}$, por lo cual se puede utilizar en el riego.
Las temperaturas de las aguas residuales de las muestras están entre $17.23^{\circ} \mathrm{C}$ a $17.99^{\circ} \mathrm{C}$ valores que superan el límite normal de los parámetros indicados por La R. M. N 323 2015 (16). Por lo tanto, no podría utilizarse en el riego. 
La Turbidez de las muestras presentan valores de 591 UNT a 1506 UNT (Unidad Nefelométrica de Turbidez), superando los límites permitidos (16). Debido a la turbidez de las aguas no pueden usarse para riego.

La acumulación de Oxígeno Disuelto (OD) en el agua tiene parámetros de 1.65 a 2.02 $\mathrm{mg} / \mathrm{L}$, indica que es muy baja, no concentra los límites permitidos para que el agua sea utilizada en riego de plantas o consumo para animales.

La conductividad eléctrica de la muestra se distribuye de 389 a $1005 \mu \mathrm{S} / \mathrm{cm}$ determinando un bajo índice de concentración al comparar los resultados con los parámetros dados por el (16). No pueden utilizarse para plantas y animales.

Los sólidos disueltos totales (SDT) concentran parámetros de 236.4 - 664.4 $\mathrm{mg} / \mathrm{L}$ demostrando que existe un bajo índice de sólidos disueltos al compararlo con la norma nacional. No siendo apta para riego ni consumo animal.

Los sólidos suspendidos totales (SST) tienen valores de 0.134 a 1.774 mg/L, demuestran que están dentro de los límites permitidos por la Organización de las Naciones Unidas para la Alimentación (16). Por este parámetro si podría ser utilizado para riego

Continuando con resultados del análisis ahora presentamos los parámetros no metálicos de las aguas residuales en la tabla 2 . Donde se incluyen, la alcalinidad, los cloruros acumulados en las aguas residuales, la dureza que presentan las aguas, Los nitratos en las muestras, la concentración de nitritos, los sulfatos, fosfatos presentes, la cantidad de amonio, la Demanda Bioquímica de Oxígeno (DBO), la acumulación de Demanda Química de Oxígeno (DQO).

Tabla 2. Parámetros inorgánicos no metálicos de las aguas residuales.

\begin{tabular}{|c|c|c|c|c|c|c|c|c|c|c|c|}
\hline \multirow[b]{2}{*}{ 䄈 } & \multirow[b]{2}{*}{ Muestra } & \multicolumn{10}{|c|}{ PARÁMETROS INORGÁNICOS NO METÁLICOS } \\
\hline & & 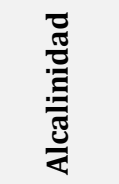 & $\begin{array}{l}n \\
0 \\
\vdots \\
0 \\
0 \\
0\end{array}$ & ปั & 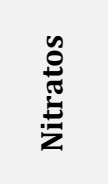 & 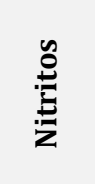 & $\frac{\mathscr{0}}{\stackrel{\pi}{\pi}}$ & $\underset{\frac{8}{\pi}}{\frac{\pi}{\pi}}$ & 茝 & 옴 & 윰 \\
\hline \multirow{5}{*}{$\begin{array}{l}\text { Colpa } \\
\text { Mayo }\end{array}$} & M1 p1 & 453,530 & 72,20 & 294,840 & 33,310 & 0.143 & 4 & 1,983 & 3.55 & 145.50 & $<0.7$ \\
\hline & M2 p2 & 307,230 & 9,98 & 115,020 & 8,973 & 0,061 & $<1,0$ & $<0,04$ & 0,55 & 65,10 & $<0,7$ \\
\hline & M3 p3 & 277,970 & 14,25 & 166,860 & $<0.1$ & 0,556 & 10 & 0,22 & 0,44 & 51,30 & 5,31 \\
\hline & M4 p4 & 248,710 & 10,45 & 158,760 & $<0,1$ & 0,677 & 1 & 1,137 & 0,70 & 98,40 & 5,22 \\
\hline & M5 p5 & 365,750 & 39,90 & 194,400 & 39,520 & 0,188 & 22 & 1,833 & 2,95 & 97,50 & $<0,7$ \\
\hline \multirow{7}{*}{$\begin{array}{c}\text { Río } \\
\text { Chotano }\end{array}$} & M6 p6 & 292,600 & 36,10 & 202,500 & 43,820 & 0,211 & 10 & 2,198 & 3,39 & 169,50 & $<0,7$ \\
\hline & M7 p7 & 380,380 & 39,90 & 217,080 & 16,610 & 0,098 & 31 & 1,727 & 1,66 & 120,40 & $<0,7$ \\
\hline & M8 p8 & 248,710 & 15,20 & 197,640 & $<0,1$ & 0,517 & 9 & 0,738 & 0,35 & 164,00 & 5,54 \\
\hline & M9 p9 & 248,710 & 8,55 & 132,840 & 9,450 & 0,176 & 6 & 0,277 & $<0,02$ & 99,20 & 7,63 \\
\hline & M10 p10 & 234,080 & 12.35 & 225,180 & 1,336 & 0,69 & 24 & 1,003 & 0,18 & 164,30 & 6,01 \\
\hline & M11 p11 & 409,640 & 63,65 & 215,460 & 51,930 & 0,226 & 27 & 1,827 & 2,18 & 161.60 & $<0,7$ \\
\hline & M12 p12 & 336,490 & 41,80 & 217,050 & 44,770 & 0,211 & 25 & 1,856 & 2,05 & 196,30 & $<0,7$ \\
\hline
\end{tabular}




\begin{tabular}{|c|c|c|c|c|c|c|c|c|c|c|c|}
\hline \multirow[b]{2}{*}{$\begin{array}{l}\frac{\pi}{\pi} \\
\frac{\pi}{0} \\
\frac{0}{0}\end{array}$} & \multirow[b]{2}{*}{ Muestra } & \multicolumn{10}{|c|}{ PARÁMETROS INORGÁNICOS NO METÁLICOS } \\
\hline & & 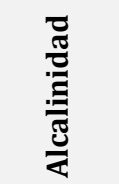 & $\begin{array}{l}\tilde{o} \\
\vdots \\
\vdots \\
0 \\
0\end{array}$ & $\begin{array}{l}\frac{\pi}{2} \\
\stackrel{\Xi}{0} \\
0\end{array}$ & 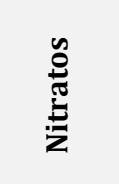 & 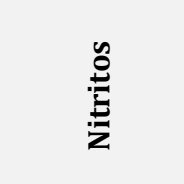 & 音 & 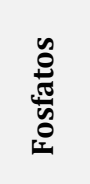 & 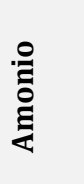 & 움 & ஜ̊̊̊ \\
\hline \multirow[t]{2}{*}{ San Juan } & M13 p13 & 365.750 & 65.55 & 213.840 & 100.100 & 0.367 & 31 & 1.985 & 3.01 & 211.40 & $<0.7$ \\
\hline & M14 p14 & 380,380 & 30,40 & 244,620 & 1,336 & 0,302 & 37 & 0.479 & 0,54 & 180,20 & 2,99 \\
\hline \multirow{3}{*}{$\begin{array}{c}\text { San } \\
\text { Mateo }\end{array}$} & M15 p15 & 468,160 & 54,15 & 260,820 & 4,200 & 0,728 & 47 & 0,347 & 0,55 & 244.80 & 2,76 \\
\hline & M16 p16 & 424,270 & 66.50 & 264.060 & 32,360 & 0,135 & 53 & 1,096 & 1,04 & 259,40 & 2,92 \\
\hline & M17 p17 & 453,530 & 37,05 & 265,680 & 32,559 & 0,135528221 & 53,32515337 & 1,103 & 1,04 & 236,60 & 2,67 \\
\hline
\end{tabular}

Fuente: Informe de ensayos 335 - 352.

Los cloruros acumulados en las aguas residuales que se desplazan por las quebradas y río tienen valores de $9.98 \mathrm{mg} / \mathrm{L}$ a $72.20 \mathrm{mg} / \mathrm{L}$ demostrando que es inferior a los límites permitidos por la norma nacional. No pueden usarse ni en riego ni para animales.

La dureza que presentan las aguas es de $115.020 \mathrm{mg} / \mathrm{L}$ y $294.840 \mathrm{mg} / \mathrm{L}$, los valores son menores a los parámetros establecidos por el MINAM (16) o DIGESA (18) para utilizarlo en el riego de plantas y bebida de animales.

Los nitratos concentrados en las muestras tienen valores de $<0.1 \mathrm{mg} / \mathrm{L} \mathrm{N}$ a $51.930 \mathrm{mg} / \mathrm{L} \mathrm{N}$, los resultados indican que el agua puede utilizarse en el riego y en animales por estar en los límites permitidos por el MINAM (16).

Las aguas residuales estudiadas concentran nitritos comprendidos entre $0.061 \mathrm{mg} / \mathrm{L} \mathrm{N} \mathrm{y}$ $0.728 \mathrm{mg} / \mathrm{L} \mathrm{N}$, valores inferiores al límite establecido por el MINAM (16).

Los análisis concentran sulfatos $<1 \mathrm{mg} / \mathrm{L} \mathrm{y}$ $53 \mathrm{mg} / \mathrm{L}$, los resultados son menores a los establecido por el MINAM (16). Por lo tanto, no se pueden usar para riego ni para animales.

Los fosfatos albergados en las muestras valores $<0.04 \mathrm{mg} / \mathrm{L}$ y $2.198 \mathrm{mg} / \mathrm{L}$, superan el límite permitido por el MINAM (16), siendo no aptas para la bebida de animales e irrigar plantas de tallo bajo y alto.

El amonio que concentran las aguas residuales tienen valores $>0.18 \mathrm{mg} / \mathrm{L}$ y 3.55 $\mathrm{mg} / \mathrm{L}$, superando el límite permitido por la norma nacional.

La Demanda Bioquímica de Oxígeno (DBO) de las muestras, tienen de $51.30 \mathrm{mg} / \mathrm{L}$ a 259 $\mathrm{mg} / \mathrm{L}$, los resultados demuestran que hay una alta concentración de DBO, es un riesgo para la salud de animales y plantas.

La acumulación de Demanda Química de Oxígeno (DQO) tienen concentraciones $<0.7$ $\mathrm{mg} / \mathrm{L}$ y $6.01 \mathrm{mg} / \mathrm{L}$, demuestran que los valores están por debajo del límite permitido por el MINAM (16). No pueden usarse para riego ni para animales.

Se presentan a continuación los análisis microbiológicos de las aguas residuales conformados por 4 grupos donde el primero formado por los coliformes con sus parámetros: dilución, Coliformes totales, Coliformes fecales y E. Coli. El segundo conformado por los estreptococos con: dilución, estreptococos fecales, enterococos fecales y finalmente se presentan Salmonela y V. Coholerae. 
Tabla 3. Análisis microbiológico de las aguas residuales.

\begin{tabular}{|c|c|c|c|c|c|c|c|c|c|c|}
\hline \multirow[b]{2}{*}{$\begin{array}{l}\frac{0}{2} \\
\frac{\pi}{2} \\
\frac{\pi}{0} \\
\frac{0}{0} \\
\frac{0}{0}\end{array}$} & \multirow[b]{2}{*}{ Muestra } & \multirow[b]{2}{*}{ } & \multicolumn{3}{|c|}{ GRUPOS COLIFORMES } & \multicolumn{3}{|c|}{ GRUPOS ESTREPTOCOCOS } & \multirow[t]{2}{*}{ SALMONELLA } & \multirow[t]{2}{*}{$\begin{array}{c}\mathrm{v} . \\
\text { COHOLERAE }\end{array}$} \\
\hline & & & 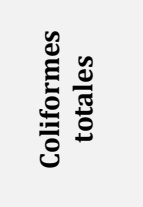 & 窇 & $\begin{array}{l}\overline{0} \\
\text { जิ }\end{array}$ & 苛 & 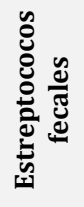 & 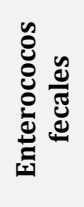 & & \\
\hline \multirow{5}{*}{$\begin{array}{l}\text { Colpa } \\
\text { Mayo }\end{array}$} & M1 p1 & 10.5 & $1600 \times 10^{5}$ & $1600 \times 10^{5}$ & $1600 \times 10^{5}$ & 10.5 & 1700 & 1400 & Presencia & Ausencia \\
\hline & M2 p2 & 10.5 & $350 \times 10^{5}$ & $350 \times 105$ & $350 \times 10^{5}$ & 10.5 & 1100 & 1100 & Ausencia & Ausencia \\
\hline & M3 p3 & 10.5 & $1600 \times 10^{5}$ & $1600 \times 10^{5}$ & $1600 \times 10^{5}$ & 10.5 & 940 & 940 & Presencia & Ausencia \\
\hline & M4 p4 & 10.5 & $1600 \times 10^{5}$ & $1600 \times 10^{5}$ & $1600 \times 10^{5}$ & 10.5 & 1700 & 1700 & Ausencia & Ausencia \\
\hline & M5 p5 & 10.5 & $1600 \times 10^{5}$ & $1600 \times 10^{5}$ & $1600 \times 10^{5}$ & 10.5 & 630 & 630 & Ausencia & Ausencia \\
\hline \multirow{7}{*}{$\begin{array}{c}\text { Río } \\
\text { Chotano }\end{array}$} & M6 p6 & 10.5 & $1600 \times 10^{5}$ & $1600 \times 10^{5}$ & $1600 \times 10^{5}$ & 10.5 & 790 & 790 & Presencia & Ausencia \\
\hline & M7 p7 & 10.5 & $920 \times 10^{5}$ & $920 \times 10^{5}$ & $920 \times 10^{5}$ & 10.5 & 460 & 460 & Ausencia & Ausencia \\
\hline & M8 p8 & 10.5 & $1600 \times 10^{5}$ & $1600 \times 10^{5}$ & $1600 \times 10^{5}$ & 10.5 & 1600 & 1600 & Presencia & Ausencia \\
\hline & M9 p9 & 10.5 & $1600 \times 10^{5}$ & $1600 \times 10^{5}$ & $1600 \times 10^{5}$ & 10.5 & 490 & 490 & Ausencia & Ausencia \\
\hline & M10 p10 & 10.5 & $1600 \times 10^{5}$ & $1600 \times 10^{5}$ & $1600 \times 10^{5}$ & 10.5 & 940 & 940 & Ausencia & Ausencia \\
\hline & M11 p11 & 10.5 & $1600 \times 10^{5}$ & $1600 \times 10^{5}$ & $1600 \times 10^{5}$ & 10.5 & 1400 & 1400 & Presencia & Ausencia \\
\hline & M12 p12 & 10.5 & $1600 \times 10^{5}$ & $1600 \times 10^{5}$ & $1600 \times 10^{5}$ & 10.5 & 940 & 940 & Ausencia & Ausencia \\
\hline \multirow{3}{*}{$\begin{array}{l}\text { San } \\
\text { Juan }\end{array}$} & M13 p13 & 10.5 & $1600 \times 10^{5}$ & $1600 \times 10^{5}$ & $1600 \times 10^{5}$ & 10.5 & 1600 & 1600 & Ausencia & Ausencia \\
\hline & M14 p14 & 10.5 & $1600 \times 10^{5}$ & $1600 \times 10^{5}$ & $1600 \times 10^{5}$ & 10.5 & 1300 & 1300 & Ausencia & Ausencia \\
\hline & M15 p15 & 10.5 & $1600 \times 10^{5}$ & $1600 \times 10^{5}$ & $1600 \times 10^{5}$ & 10.5 & 1700 & 1700 & Presencia & Ausencia \\
\hline \multirow[t]{2}{*}{$\begin{array}{c}\text { San } \\
\text { Mateo }\end{array}$} & M16 p16 & 10.5 & $1600 \times 10^{5}$ & $1600 \times 10^{5}$ & $1600 \times 10^{5}$ & 10.5 & 700 & 700 & Presencia & Ausencia \\
\hline & M17 p17 & 10.5 & $1600 \times 10^{5}$ & $1600 \times 10^{5}$ & $1600 \times 10^{5}$ & 10.5 & 1600 & 1600 & Ausencia & Ausencia \\
\hline
\end{tabular}

Fuente: Informe de ensayos 335 - 352.

El análisis microbiológico de las muestras presenta dilución de $10.5 \mathrm{ml}$, el Número más Probable (NMP) de coliformes totales en las aguas residuales es de $350 \times 10^{5}$ a $1600 \times 10^{5}$, que indican parámetros sumamente altos que superan los estándares permitidas del MINAM (16).

Los coliformes fecales de las muestras presentan valores de $350 \times 10^{5}$ a $1600 \times 10^{5}$, representa de $35000000 \mathrm{NMP} / 100 \mathrm{ml} \mathrm{a}$ $160000000 \mathrm{NMP} / 100 \mathrm{ml}$, demuestran que existe una alta concentración de coliformes fecales, las aguas residuales no pudiéndose utilizar en el riego de la producción agrícola.
La concentración de EscherichiaColi, de las muestra tienen de $350 \times 10^{5}$ a $1600 \times 10^{5}$, representa $35000000 \mathrm{NMP} / 100 \mathrm{ml} \quad \mathrm{a}$ $160000000 \mathrm{NMP} / 100 \mathrm{ml}$, los resultados demuestran que hay una alta concentración y que no pudiéndose utilizar en las actividades agrícolas. (16).

En una dilución de $10.5 \mathrm{ml}$ de agua las muestras tienen acumulaciones de 460 estreptococos fecales/100 $\mathrm{ml}$ a $2100 / 100 \mathrm{ml}$, conteniendo alta concentración de esta bacteria y que las aguas están contaminadas.

La concentración de enterococos fecales en las muestras es de $460 \mathrm{NMP} / 100 \mathrm{ml} \mathrm{a}$ 
$2100 \mathrm{NMP} / 100 \mathrm{ml}$, implica un riesgo para la salud.

El análisis micro biológico de las aguas residuales indica que en las muestras $1,3,6$, $8,11,15$ y 16 existe presencia de salmonella, mientras que en el resto de las muestras está ausente. Lo cual la hace no apta para consumo.
El análisis microbiológico de las aguas residuales indica que hay ausencia del Vibrio Cholerae en las muestras. Pudiendo entonces usarse para riego y para animales.

Seguidamente se muestra los análisis microbiológicos de la producción agrícola consistentes en repollo, papa, racacha, lechuga, acelga, cebolla y berenjena.

Tabla 4. Análisis microbiológico de la producción agrícola.

\begin{tabular}{|c|c|c|c|c|c|c|}
\hline \multicolumn{2}{|c|}{$\begin{array}{c}\text { Unidad de Medida } \\
\text { Muestras }\end{array}$} & \multirow{2}{*}{$\begin{array}{c}\mathrm{N}^{\circ} \text { de } \\
\text { Coliformes } \\
\text { totales } \\
\text { NMP/g } \\
\end{array}$} & \multirow{2}{*}{$\begin{array}{c}\mathrm{N}^{\circ} \text { de } \\
\text { Coliformes } \\
\text { fecales } \\
\text { NMP/g } \\
\end{array}$} & \multirow{2}{*}{$\begin{array}{c}\text { Detección de } \\
\text { Enterobacterias } \\
\text { Ausencia/ } \\
\text { Presencia } \\
\end{array}$} & $\begin{array}{c}\text { Detección de } \\
\text { Salmonella sp } \\
\text { Ausencia/ } \\
\text { Presencia } \\
\end{array}$ & $\begin{array}{c}\mathbf{N}^{\circ} \text { de } \\
\text { Aerobios } \\
\text { mesófilos } \\
\text { UFC/g }\end{array}$ \\
\hline \multirow{4}{*}{ Repollo } & & & & & & \\
\hline & M1 & $>1100$ & $>1100$ & Presencia & Presencia & Incontable \\
\hline & M2 & $>1100$ & $>1100$ & Presencia & Ausencia & Incontable \\
\hline & M7 & $>1100$ & $>1100$ & Presencia & Ausencia & Incontable \\
\hline \multirow{3}{*}{ Papa } & M1 & 1100 & 1100 & Presencia & Ausencia & $25 \times 10^{5}$ \\
\hline & M2 Cascara & $>1100$ & $>1100$ & Presencia & Ausencia & Incontable \\
\hline & M3 Cascara & $>1100$ & $>1100$ & Presencia & Ausencia & Incontable \\
\hline \multirow{3}{*}{ Racacha } & M4 Almidón & 1100 & 1100 & Presencia & Ausencia & $36 \times 10^{5}$ \\
\hline & M1 & $>1100$ & $>1100$ & Presencia & Presencia & Incontable \\
\hline & M2 Almidón & 1100 & 500 & Presencia & Presencia & $11 \times 10^{4}$ \\
\hline Lechuga & M4 P7 & $>1100$ & $>1100$ & Presencia & Ausencia & Incontable \\
\hline \multirow{2}{*}{ Acelga } & M1 & $>1100$ & $>1100$ & Presencia & Presencia & Incontable \\
\hline & M2 & $>1100$ & $>1100$ & Presencia & Ausencia & Incontable \\
\hline Cebolla & M1 & $>1100$ & $>1100$ & Presencia & Presencia & Incontable \\
\hline \multirow{2}{*}{ Berenjena } & M1 Pulpa & 150 & 21 & Ausencia & Ausencia & $8 \times 10^{4}$ \\
\hline & M2 Cascara & $>1100$ & $>1100$ & Presencia & Ausencia & $11 \times 10^{4}$ \\
\hline
\end{tabular}

Fuente: Informe de Ensayos de Muestras.

Los análisis microbiológicos de las muestras presentaron los siguientes resultados:

Repollo (tres muestras): Las muestras que acumulan coliformes totales y fecales $>1000$, implica que es un alimento inaceptable para el consumo humano, presentan además Enterobacterias. La muestra 1 tiene presencia de salmonella y las otras 2 ausencia, el número de Aerobios mesófilos son incontables.

Papa (cuatro muestras): según el método de Número Más Probable (NMP) de las muestras concentran presencia de coliformes totales y fecales entre 1100 y $>1100$, la presencia de enterobacterias en la cascara y almidón, ausencia de salmonella y el número de aerobios mesófilos $25 \times 10^{5}$ en la muestra 1 
y $36 \times 10^{5}$ en la muestra 4, siendo incontables en las muestras 2 y 3 .

Racacha (dos muestras): indica que hay presencia de coliformes totales entre 1100 $\mathrm{NMP} / \mathrm{g}$ y $>1100 \mathrm{NMP} / \mathrm{g}$, coliformes fecales entre $500 \mathrm{NMP} / \mathrm{g}$ y $>1100 \mathrm{NMP} / \mathrm{g}$, presencia de enterobacterias y el número de aerobio mesófilos es incontable en la primera muestra y $11 \times 10^{4}$ en la segunda muestra, los resultados están lejos de los parámetros de la norma plantea por DIGESA (18).

Lechuga (una muestra): acumula coliformes totales $>1100$ y la misma cantidad de coliformes fecales, tiene presencia de enterobacterias y número incontable de aerobios mesófilos, los resultados demuestran que la lechuga que se produce en el valle del río Chotano no se puede consumir en ensaladas.

Acelga (dos muestras): Contienen coliformes totales y fecales $>1100$, presencia de enterobacterias en las 2 muestras, salmonella en la M1 y aerobios mesófilos incontables.

Cebolla (una muestra): tiene presencia de coliformes totales y fecales $>1100 \mathrm{NMP} / \mathrm{g}$, presencia de enterobacterias y salmonella, número incontable de aerobios mesófilos, los resultados demuestran que la cebolla está contaminada.

Berenjena (pulpa y cascara): presenta coliformes totales de 150NMP/g y $>1100 \mathrm{NMP} / \mathrm{g}$, coliformes fecales de 21 $\mathrm{NMP} / \mathrm{g}$ y $>1100 \mathrm{NMP} / \mathrm{g}$, en la cascara contiene enterobacterias y aerobios mesófilos de $8 \times 10^{4}$ y $11 \times 10^{4}$, los resultados demuestran que afectaría la salud de sus consumidores.

\section{CONCLUSIONES}

Se lograron identificar los parámetros físicos y de agregación para las aguas residuales sin tratamiento de quebradas San Mateo, Colpamayo, San Juan y Río Chotano provenientes de la ciudad de Chota, donde se establece que solo dos parámetros: el $\mathrm{pH}$ y los sólidos en suspendidos totales están entre los valores adecuados. La temperatura y la turbidez están fuera de rango por exceso en sus valores y el oxígeno disuelto, la conductividad eléctrica, los sólidos disueltos totales están fuera de rango por no alcanzar el mínimo valor.

Entre los parámetros inorgánicos no metálicos que están en los valores aceptables están: la alcalinidad, los cloruros, la dureza, los nitratos, los nitritos, los sulfatos y la demanda química de oxígeno y quienes no están en los valores correctos son: los fosfatos, el amonio, y la demanda química de oxígeno.

Entre los agentes contaminantes de las aguas residuales encontrados en las muestras se tienen: Coliformes totales, coliformes fecales, estreptococos fecales, enterococos fecales, salmonella, ninguna muestra contuvo V. coholerae.

Se determinó la acumulación de agentes contaminantes en la producción agrícola por el uso de las aguas residuales. Los productos analizados: Repollo, Papa, Racacha, Lechuga, Acelga, Cebolla y Berenjena presentaron contaminación por uno o varios de entre: coliformes fecales, coliformes totales, enterobacterias, salmonella $\mathrm{o}$ aerobios mesófilos. Los que los hace no aptos para el consumo humano.

No es aceptable el riego de productos agrícolas ni el consumo por animales de aguas residuales si estas no son previamente tratadas. Porque podía convertirse este consumo en un problema de salud pública, al consumir alimentos contaminados. 
REFERENCIAS

1. Winpenny J, Heinz I, Koo S. Reutilizacion del agua en la agricultura: ¿Beneficios para todos? 2013. (FAO, Editor) Recuperado el 10 de marzo de 2021, de http://www.fao.org/3/i1629s/i1629s.pdf.

2. Zamora F, Rodriguez N, Torres D, Yendis. Efecto del riego con aguas residuales sobre las propiedades quimicas de los suelos de la planicie de Coro, estado Falcón. Bioagro. 2008. 20 (3), 193-199.

3. Peña M, Ducci J, Zamora V. Tratamiento de Aguas Residuales en México. 2013. Recuperado el 10 de Marzo de 2021, de http://www.siagua.org/sites/default/files/do cumentos/documentos/tratamiento_de_agua s_residuales_en_mexico.pdf.

4. Ministerio de Agricultura. Autoridad Nacional del Agua. Autoridad Administrativa del agua Cañete - Fortaleza. Administración Local de Agua Chillón - Rímac - Lurin. (A. N. Agua, Editor, A. A. Fortaleza, Productor, \& Administradora local de Agua Chillón- RímacLurin). 2015. Recuperado el 10 de marzo de 2021, de repositorio.ana.gob.pe/bitstream/handle/AN A/2456/ANA0001215.pdf.

5. Noticias Ser. Cajamarca: La otra contaminación. 2015. Recuperado el 11 de Marzo de 2021, de http://prevencionar.com.pe/2015/06/04/caj amarca-la-otra-contaminacion/

6. La República. Cajamarca: Minera Yanacocha vertió 40 millones de metros cúbicos de agua residual al ambiente. 2016. Recuperado el 10 de Marzo de 2021, de http://larepublica.pe/turismo/rumbos-aldia/812777-cajamarca-minera-yanacochavertio-40-millones-de-metros-cubicos-deagua-residual-al-ambiente
7. Forsi, C. El uso de las aguas residuales tratadas en Mendoza - Argentina. (\&. R. En H. Hettiarachchi, Ed.) Uso seguro de aguas residuales en la agricultura: ejemplo de buenas prácticas. 2017. 261 - 277.

8. Guadarrama, M., \& Galván, A. Impacto del uso de agua residual en la agricultura. Iberoamericana de Ciencias Biológicas y Agropecuarias. 2015. 1-23.

9. Mendoza, M. A. Evaluación fisicoquímica de la calidad del agua superficial en el Centro Poblado de Sacsamarca, Región Ayacucho. 2018. Lima: Trabajo especial de grado de la Pontificia Universidad Católica del Perú.

10. Tarrillo, C. P. Rehabilitación, ampliación y unificación de las plantas de tratamiento de aguas residuales del sector noroeste de Piura. 2017. Lima: Trabajo especial de grado de la Universidad Nacional de Ingeniería.

11. Reyes, C. M. Estudio de contaminación de las aguas del río Chillón. 2012. Lima: Trabajo especial de grado de la Universidad Nacional de Ingeniería.

12. Galindo, F., Jacobo, M., Cueto, J., \& Reta, S. Caracterización de aguas residuales tratadas de la comarca lagunera y su viabilidad en el riego agrícola. 2020. Revista Mexicana de Ciencias Agrícolas, 11 (1), 189-201.

13. OMS. Guías para la calidad del agua potable. 2017.

14. Organización Mundial de la Salud. Inocuidad de los Alimentos. 2020. (OMS, Ed.) Recuperado el 11 de Marzo de 2021, de https://www.who.int/es/news-room/factsheets/detail/food-safety

15. FAO. El manejo del suelo en la producción de hortalizas con buenas prácticas agrícolas. 2013. (O. d. Agricultura., Editor) Recuperado 
el 10 de marzo de 2021, de Agua. Recuperado el 11 de Marzo de 2021, de http://www.fao.org/3/a-i3361s.pdf.

16. MINAM. Resolución Ministerial $N^{\circ} 323$ 2015-MINAM. 2015. Recuperado el 11 de

Marzo de 2021, de
https://www.minam.gob.pe/disposiciones/re solucion-ministerial-n-323-2015-minam/

17. Dirección General de Salud Ambiental. (s.f). Estándares de Calidad Ambiental de http://www.digesa.minsa.gob.pe/DEPA/infor mes_tecnicos/GRUPO\%20DE\%20USO\%203.p df.

18. DIGESA. (s.f). Parámetros Organolépticos. Recuperado el 11 de marzo de 2021, de http://www.digesa.minsa.gob.pe/DEPA/infor mes_tecnicos/GRUPO\%20DE\%20USO\%201.p df. 